PAPER

\title{
Brain activation to Japanese words spoken with incorrect pitch accent
}

\author{
Ikuyo Masuda-Katsuse* \\ Faculty of Humanity-Oriented Science and Engineering, Kinki University, \\ 11-6, Kayanomori, Iizuka, 820-8555 Japan
}

(Received 3 February 2010, Accepted for publication 5 October 2010)

\begin{abstract}
In this study, brain activation when listening to spoken Japanese with incorrect pitch accents was investigated. In the event-related fMRI experiment, four kinds of stimuli were prepared. (1) NORMAL: Real words with a correct pitch accent. (2) INCORRECT: Real words with incorrect pitch accent. (3) PSEUDO: Pseudo-words, and (4) BASE: amplitude-modulated pseudo-speech noise. Fifteen normal right-handed participants were scanned by functional MRI while listening to these stimuli. Region of interest (ROI) analyses were performed. The contrast between INCORRECT and NORMAL revealed significant activation in the bilateral inferior frontal, the bilateral precentral, and the left supplementary motor area, and the right superior temporal gyrus. The result supports the view that speech perception is a sensory-motor process, and suggests that when the pitch accent of the stimulus does not match the template, silent rehearsal might be repeated to enable a lexical decision to be made.
\end{abstract}

Keywords: Speech perception, fMRI, Pitch accent language, Auditory-motor interaction

PACS number: 43.64.Sj, 43.71.An [doi:10.1250/ast.32.62]

\section{INTRODUCTION}

Japanese is one of the pitch-accent languages, in which high or low pitch is determined at every mora, and there is a carrier of accent on the mora just before the mora where the pitch drops to a lower level. In a pitch-accent language, pitch height at every mora of spoken words is of little importance in comparison with tonal languages such as Mandarin Chinese. In Japanese, where the pitch falls is important, whereas in Mandarin Chinese, how pitch rises and falls is important. Thus, the height of speech sound acts differently between languages, although most languages use pitch patterns to achieve individual word meaning [1].

Masuda-Katsuse [2] examined the contribution of pitch-accent information to Japanese spoken-word recognition. Using speech stimuli to manipulate pitch accents, she conducted a word intelligibility test under different signal-to-noise ratio conditions and a rating test for the adequacy of accent types in words. The word intelligibility scores were examined on the basis of the adequacy rating of the accent types. Results revealed that under noisy conditions, the higher the adequacy of the accent type was rated, the higher the word intelligibility was. Under the

*e-mail: katsuse@fuk.kindai.ac.jp clean condition, the word intelligibility was almost $100 \%$ even when the accent type was inadequate for the word. That is to say, phonetic information is sufficient to identify the word. Moreover, she measured the latency in shadowing under the clean condition. The participants were presented speech stimuli with manipulated accent types and shadowed the words as quickly as possible. The results indicated a significant negative correlation between latency and the adequacy rating of accent types. In the case of a word with inadequate pitch accent, additional processing might cause a stretch of latency even if sufficient phonetic information were obtained for word identification. Then, what is the additional processing?

The objective of this study is to reveal how the brain activity when participants listen to Japanese spoken words with an incorrect pitch accent is different from that for words with correct pitch accent.

\section{MATERIALS}

\subsection{Word List}

Japanese four-mora words were used in the experiments. These have four standard types of accent. Each accent type is represented by high-and-low patterns of pitch as follows: low-high-high-high for accent type 0 , high-low-low-low for accent type 1, low-high-low-low for accent type 2, and low-high-high-low for accent type 3 . 
Four-mora words were selected from the database included in Lexical Properties of Japanese [3] on the basis of the following criteria: their familiarity is 6.0 or more in auditory presentation, their familiarity is 5.0 or more in visual presentation, and their familiarity is 5.0 or more in both auditory and visual presentation. The maximum value of the familiarity is 7 .

By selecting 50 words randomly from among words of each accent type, 200 words were listed. In addition, fourmora nonsense words were generated by randomly arranging the syllables appearing in the 200 words.

\subsection{Speech Materials}

Speech materials were generated in a similar manner to that in the previous study [2]. A male speaker read the word lists in a soundproof booth and the speech sounds were recorded on digital audio tape. The speaker was instructed to read the lists monotonously and at a fixed speed and on beat at each mora.

First, the recorded speech sounds were analyzed using the STRAIGHT [4]. STRAIGHT enables high-quality speech resynthesis even when the pitch, the speaking rate and the spectral shape of the speech are greatly changed. In STRAIGHT synthesis, it is assumed that the combined vocal tract and glottal pulse response is in a minimum phase. The vocal tract can be modeled as a linear timeinvariant system, the output of which is the convolution of the impulse response of the vocal tract with the excitation waveform [5]. The transfer function of the vocal tract can be calculated from complex cepstra, which can be calculated from the amplitude spectrum obtained by STRAIGHT analysis. The glottal pulse is generated by an all-pass filter design [4], in which the characteristic of the temporal energy distribution of the excitation sound source is controlled by manipulating the group delay characteristic.

Thus, speech stimuli were resynthesized using STRAIGHT by transforming the original $F_{0}$ into an alternative $F_{0}$ while normalizing the speech duration to $850 \mathrm{~ms}$. The alternative $F_{0}$ was generated by adding a small $F_{0}$ fluctuation, which was obtained by eliminating a trend component from the original $F_{0}$, to one of the four representative $F_{0}$ contours. Thus, four kinds of stimuli were generated for every word of the four accent types: $0,1,2$, and 3.

The amplitude of the stimuli was normalized by the maximum power among them in order to play the stimuli as loud as possible in scanner noise.

\subsection{Selection of Stimuli for the fMRI Experiment}

In the fMRI experiment, four kinds of stimuli were prepared.

(1) NORMAL: Real words with correct pitch accent.
(2) INCORRECT: Real words with incorrect pitch accent.

(3) PSEUDO: Pseudo-words.

(4) BASE: Amplitude-modulated pseudo-speech noise.

In Japanese, there is a standard accent (that is, Kanto accent) for speech. Although we can regard a stimulus with such a standard accent as NORMAL, it is difficult to define an incorrect accent for such a word. Various accent types are used in practice, depending on the dialect. Nowadays, with the advent of media such as television and the active movement of people from place to place, most participants may have heard dialects other than the Kanto dialect. To avoid such problems, in the previous study [2], a new subjective scale was introduced for evaluating the adequacy of an accent type of a word. When a word was presented as an auditory stimulus, participants rated the adequacy of the word's accent. They did not rate the accent based on whether it was a Kanto accent or not, but rather on another standard that was formed based on the participants' linguistic experience. If a word has a low average of the rating values for an accent type, there is a high probability that many Japanese people will evaluate the accent type as incorrect for that word.

Therefore, in this study, NORMAL and INCORRECT stimuli were selected on the basis of the evaluation values obtained in the previous study [2]. In ascending order of the average of rating scores, 60 stimuli were selected as NORMAL. In descending order of the average of rating scores, 60 stimuli were selected as INCORRECT. If a selected word duplicated one in the NORMAL category, that word was removed.

For the PSEUDO condition, 30 four-mora pseudowords were selected from nonsense words that were generated by arranging four syllables randomly selected from all syllables present in the words in the NORMAL and INCORRECT categories.

For the BASE condition, amplitude-modulated pseudospeech noise was generated. The frequency characteristic shows the average frequency characteristics of male and female speech sounds, presented by Dunn and White [6]. Amplitude contours were extracted from the speech stimuli in NORMAL, INCORRECT, and PSEUDO conditions. The base stimuli were generated from amplitude-modulating pseudo-speech noise with these amplitude contours. Such noise has a similar spectrum and time-variation of amplitudes to those of the speech stimuli but do not contain pitch or phonetic information.

Table 1 shows several examples of the words used in NORMAL, INCORRECT, and PSEUDO conditions.

\section{PARTICIPANTS}

The fifteen participants ( 8 males and 7 females; ranging from 21-46 years old) were native Japanese speakers, right-handed, and had not experienced any hearing disor- 
Table 1 Examples of words used in the experiment.

\begin{tabular}{|c|c|c|c|}
\hline condition & word examples & meaning & $\begin{array}{c}\text { accent } \\
\text { type }\end{array}$ \\
\hline \multicolumn{4}{|l|}{ NORMAL } \\
\hline & Têburu & table & type 0 \\
\hline & Tôtaru & total & type 1 \\
\hline & Nomimizu & drinking water & type 2 \\
\hline & Hanareru & leave & type 3 \\
\hline \multicolumn{4}{|l|}{ INCORRECT } \\
\hline & Nîsan & brother & type 0 \\
\hline & Nenmatu & the end of the year & type 1 \\
\hline & Undô & exercise & type 2 \\
\hline & Saboten & cactus & type 3 \\
\hline \multicolumn{4}{|l|}{ PSEUDO } \\
\hline & Sirudowa & & type 0 \\
\hline & Totosato & & type 1 \\
\hline & Remuhiro & & type 2 \\
\hline & Imosimi & & type 3 \\
\hline
\end{tabular}

der. All participants had participated in other fMRI experiments but had not participated in the rating test of the adequacy of pitch accent of the words. The safety and ethics of the experiment were examined beforehand by the ATR Review Board Ethics Committee and the ATR Review Board Safety Committee for Functional Resonance Imaging Research and were approved. All participants gave written informed consent that was approved by both committees.

\section{PROCEDURE}

\subsection{Scanning Procedure}

The experiment consisted of two sessions. One experimental session consisted of 105 trials. The stimuli were allocated at random into each session in a random order. Between sessions, the participants were given a short break, but they were not permitted to move their bodies. The interval between stimuli was alternated between $4.0 \mathrm{~s}$ and $5.5 \mathrm{~s}$. The participants lay supine inside the scanner with their eyes closed and wore closed earphones. All auditory stimulation was delivered binaurally. The participants held a switch box in their left hand (opposite their dominant hands) and were asked to respond by pushing button 1 when they judged the stimulus to be a real word or button 2 when they judged the stimulus to be a nonsense word or noise.

Before the experiment, the participants practiced pushing the buttons after listening to the stimuli from a different word set. In this practice session, the participants controlled the sound level so as to hear clearly even in the presence of scanner noise. The sound pressure levels were about $80 \mathrm{~dB}$ (A), although they varied slightly depending on the participant.
Table 2 Correct rates of button responses.

\begin{tabular}{cc}
\hline condition & $\begin{array}{c}\text { correct rate of } \\
\text { button responses }(\%)\end{array}$ \\
\hline NORMAL & 97.0 \\
INCORRECT & 90.6 \\
PSEUDO & 97.3 \\
NOISE & 99.7 \\
\hline
\end{tabular}

\subsection{Effect of Scanner Noise on Speech Perception}

Scanner noise occurs in the fMRI scanner. The noise might interfere with the participants' listening ability although they wore closed earphones. Here, we confirm whether the interference was serious or not. The participants were required to answer whether each word was real or not by pushing the appropriate button. Even though the correct rate of button responses does not directly reflect the intelligibility, it can provide a rough assessment of the effect of noise.

Table 2 shows correct rates of button responses. All correct rates were sufficiently high to ensure the reliability of the results of the experiment for the objective of this study. The disturbance seemed to be slight even when the participants' listening ability was disturbed by the scanner noise.

\section{IMAGE ACQUISITION AND PREPROCESSING}

Scanning was performed using Shimadzu-Marconi Magnex Eclipse 1.5 T Power Drive 250 at the ATR Brain Activity Imaging Center. Functional T2*-weighted images were acquired using a gradient echo-planar imaging sequence. TR was $3,000 \mathrm{~ms}$, the thickness was $5 \mathrm{~mm}(z$ direction), and the in-plane resolution was $3 \times 3 \mathrm{~mm}(x, y$ directions). The whole brain was scanned. Images were preprocessed using programs within Statistical Parametric Mapping 2002 (SPM2, Wellcome Department of Imaging Neuroscience, University College London, London, UK). The images were realigned to revise participants' head movements, normalized spatially to the MNI standard brain, and smoothed spatially. The full-width at halfmaximum (FWHM) of the spatial filter was twice as long as the voxel resolution.

\section{STATISTICAL ANALYSIS}

Data were analyzed with SPM2. For two out of fifteen participants, the movements of their head position during the experiments were more than half the voxel resolution (that is, $1.5 \mathrm{~mm}$ ). In this case, the obtained coordinates of active areas were shifted from the real positions because of such movements, so all data from them were discarded. As a result, image data of thirteen participants were statistically analyzed. 
In advance, whole brain activation was observed for NORMAL, INCORRECT, and PSEUDO conditions in contrast to the BASE condition. The brain activation revealed by INCORRECT or PSEUDO vs BASE is stronger than that revealed by NORMAL vs BASE. Therefore, two kinds of contrast analyses, between INCORRECT and NORMAL and between PSEUDO and NORMAL, were performed for each participant. In addition, a contrast analysis between NORMAL and BASE was performed. Then, region of interest (ROI) analyses of random effect models were performed.

\subsection{ROI Selection}

Some hypotheses were assumed as a result of the two contrasts in the current study. Six ROIs were selected by considering previous studies related to the hypotheses.

First, we consider the contrast between PSEUDO and NORMAL. There are several previous studies on the investigation of the differences in brain activation between real and pseudo words. As a result of the contrast between normal and pseudo words, significant activation has been reported in the inferior frontal gyrus (IFG) [7,8]. As a result of the contrast between pseudo and normal words, significant activation has been reported in the IFG [9-12], the superior temporal gyrus (STG) [9], and the supplementary motor area (SMA) [10,11].

For the contrast between INCORRECT and NORMAL, real words were used in both conditions. What is different between them?

One difference is familiarity (or frequency). The stimuli in the NORMAL condition were familiar to the participants, on the other hand, those in the INCORRECT condition were unusual for the participants. Several previous studies have revealed the effects of word frequency on brain activation. Significant activation has been observed in the SMA [12,13], pre-SMA [13] and IFG [14].

From another point of view, the mismatch between the pitch accent of the stimuli and the normal of the word may cause conflicting detection. Many studies have shown that the anterior cingulate cortex (ACC) serves as a conflict detector [15-18]. Moreover, a process to solve the mismatch will be required. This process may be similar to the forward model proposed by Iacoboni [19]. His model accounted for the results of the experiments obtained by Wilson and Iacoboni [20] which revealed neural responses to unfamiliar non-native phonemes. According to his model, when a match between input and template phonemes is never obtained, the motor system is engaged in repeated attempts to generate other internal auditory templates, leading to greater motor activity in the superior part of the ventral premotor area (PMsv).

Another difference between INCORRECT and NORMAL may be found in prosody processing. The areas
Table 3 ROI definition.

\begin{tabular}{|c|c|c|}
\hline ROI & side & $\begin{array}{l}\text { coordinates } \\
(\mathrm{x}),(\mathrm{y}),(\mathrm{z})\end{array}$ \\
\hline $\begin{array}{l}\text { orbital part } \\
\text { of IFG }\end{array}$ & $\begin{array}{l}\mathrm{L} \\
\mathrm{R}\end{array}$ & $\begin{array}{c}(-54,-14),(8,54),(-26,-2) \\
(16,62),(12,50),(-26,-2)\end{array}$ \\
\hline $\begin{array}{l}\text { opercular part } \\
\text { of IFG }\end{array}$ & $\begin{array}{l}\mathrm{L} \\
\mathrm{R}\end{array}$ & $\begin{array}{c}(-64,-32),(0,26),(0,34) \\
(30,66),(2,24),(-2,38)\end{array}$ \\
\hline $\begin{array}{l}\text { triangular part } \\
\text { of IFG }\end{array}$ & $\begin{array}{l}\mathrm{L} \\
\mathrm{R}\end{array}$ & $\begin{array}{c}(-60,-30),(10,48),(-2,30) \\
(30,66),(12,50),(-2,30)\end{array}$ \\
\hline $\begin{array}{l}\text { superior motor } \\
\text { area (SMA) }\end{array}$ & $\begin{array}{l}\mathrm{L} \\
\mathrm{R}\end{array}$ & $\begin{array}{c}(-18,2),(-22,30),(44,78) \\
(0,18),(-30,26),(46,78)\end{array}$ \\
\hline pre-SMA & $\begin{array}{l}\mathrm{L} \\
\mathrm{R}\end{array}$ & $\begin{array}{l}(-20,0),(0,30),(50,80) \\
(20,0),(0,30),(-50,80)\end{array}$ \\
\hline $\begin{array}{l}\text { superior } \\
\text { temporal } \\
\text { gyrus } \\
\text { (STG) }\end{array}$ & $\begin{array}{l}\mathrm{L} \\
\mathrm{R}\end{array}$ & $\begin{array}{c}(-68,-36),(-54,6),(-16,22) \\
(36,72),(-60,4),(-16,22)\end{array}$ \\
\hline $\begin{array}{l}\text { anterior } \\
\text { cingulate } \\
\text { cortex } \\
(\mathrm{ACC})\end{array}$ & $\begin{array}{l}\mathrm{L} \\
\mathrm{R}\end{array}$ & $\begin{array}{l}(-20,0),(5,35),(10,40) \\
(0,20),(5,35),(10,40)\end{array}$ \\
\hline $\begin{array}{l}\text { middle } \\
\text { temporal } \\
\text { gyrus (MTG) }\end{array}$ & $\begin{array}{l}\mathrm{L} \\
\mathrm{R}\end{array}$ & $\begin{array}{c}(-72,-36),(-76,10),(-34,22) \\
(38,72),(-78,8),(-32,22)\end{array}$ \\
\hline
\end{tabular}

concerned with sentence-level prosody processing have been reported to be the STG, middle frontal gyrus (MFG), and medial frontal including SMA [21-23]. The STG, MFG and IFG have been reported to be involved in prosody generation [24,25].

Thus, by considering the result of previous studies, six areas, IFG (orbital, opercular, and triangular), STG, SMA (SMA proper and pre-SMA), ACC, MTG, and PMsv, were finally selected as our ROIs.

\subsection{Results}

ROI analyses were performed using the MarsBaR ROI toolbox [26]. The regions of the IFG, SMA-proper, MTG, and STG were defined using the MarsBaR ROI toolbox. The regions of the pre-SMA and ACC were defined on the basis of a reference paper [27]. The coordinates of the regions are shown in Table 3. The region of the PMsv was defined as a box region with an edge length of was $30 \mathrm{~mm}$ and a center at $(-50,-6,47)$ for the left side and $(50,6,47)$ for the right side. The central coordinates of PMsv were cited from the reference paper [20].

No significant activation was shown in the bilateral ACC and MTG for both conditions. For the INCORRECT condition compared with the NORMAL condition, significant activations were shown in the bilateral IFG, left SMA, left pre-SMA, bilateral PMsv, and right STG. For the PSEUDO condition compared with the NORMAL condition, significant activations were shown in the right IFG 
Table 4 Significant activation foci for each contrast obtained in ROI analysis $\left(^{*}: p<0.05,{ }^{* *}: p<0.01\right)$.

\begin{tabular}{|c|c|c|c|c|}
\hline ROI & contrast & side & $\begin{array}{c}\text { contrast value } \\
\text { (\% signal change) }\end{array}$ & t statistic \\
\hline \multirow{4}{*}{$\begin{array}{l}\text { orbital part } \\
\text { of IFG }\end{array}$} & $\mathrm{I}-\mathrm{N}$ & $\mathrm{L}$ & 0.87 & $3.02^{* *}$ \\
\hline & & $\mathrm{R}$ & 0.86 & $3.32^{* *}$ \\
\hline & P-N & $\mathrm{R}$ & 0.63 & $2.61^{*}$ \\
\hline & N-B & $\mathrm{L}$ & 0.60 & $2.95^{* *}$ \\
\hline \multirow{3}{*}{$\begin{array}{l}\text { opercular part } \\
\text { of IFG }\end{array}$} & $\mathrm{I}-\mathrm{N}$ & $\mathrm{L}$ & 0.75 & $2.30^{*}$ \\
\hline & & $\mathrm{R}$ & 0.55 & $3.33^{* *}$ \\
\hline & P-N & $\mathrm{R}$ & 0.52 & $2.62^{*}$ \\
\hline \multirow{4}{*}{$\begin{array}{l}\text { triangular part } \\
\text { of IFG }\end{array}$} & $\mathrm{I}-\mathrm{N}$ & $\mathrm{L}$ & 0.63 & $2.59^{*}$ \\
\hline & & $\mathrm{R}$ & 0.58 & $2.67^{*}$ \\
\hline & $\mathrm{P}-\mathrm{N}$ & $\mathrm{R}$ & 0.60 & $2.78^{* *}$ \\
\hline & N-B & $\mathrm{L}$ & 0.68 & $2.32^{* *}$ \\
\hline $\begin{array}{l}\text { superior motor } \\
\text { area (SMA) }\end{array}$ & $\mathrm{I}-\mathrm{N}$ & $\mathrm{L}$ & 0.48 & $1.99^{*}$ \\
\hline \multirow[t]{2}{*}{ pre-SMA } & $\mathrm{I}-\mathrm{N}$ & $\mathrm{L}$ & 0.47 & $2.03^{*}$ \\
\hline & $\mathrm{N}-\mathrm{B}$ & $\mathrm{L}$ & 0.37 & $1.81^{*}$ \\
\hline \multirow{3}{*}{$\begin{array}{l}\text { superior part } \\
\text { of ventral } \\
\text { premotor } \\
(\mathrm{PMsv})\end{array}$} & $\mathrm{I}-\mathrm{N}$ & $\mathrm{L}$ & 0.41 & $1.93^{*}$ \\
\hline & & $\mathrm{R}$ & 0.32 & $1.78^{*}$ \\
\hline & $\mathrm{P}-\mathrm{N}$ & $\mathrm{R}$ & 0.40 & $2.20^{*}$ \\
\hline $\begin{array}{l}\text { superior } \\
\text { temporal } \\
\text { gyrus (STG) }\end{array}$ & $\mathrm{I}-\mathrm{N}$ & $\mathrm{R}$ & 0.25 & $1.90^{*}$ \\
\hline
\end{tabular}

I-N: INCORRECT compared to NORMAL,

P-N: PSEUDO compared to NORMAL,

N-B: NORMAL compared to BASE

and right PMsv. Table 4 shows significant activation foci for each contrast obtained in ROI analysis.

\section{DISCUSSION}

\subsection{Inferior Frontal Gyrus}

In the INCORRECT condition compared with the NORMAL condition, significant activation was observed bilaterally. On the other hand, significant activation was observed only on the right side in the PSEUDO condition compared with the NORMAL condition.

The left IFG (particularly the orbitaris and triangular part) was considered to be involved in semantic processing [28]. Therefore, no significant activation in the left IFG seemed to be observed in the PSEUDO condition compared with the NORMAL condition. Then, why is significant activation observed in the left IFG in the INCORRECT condition compared with the NORMAL condition? Nakic et al. [14] showed greater activity in the bilateral IFG when participants made a lexical decision concerning lowfrequency rather than high-frequency words. They concluded that semantic representations weakly activated by a low-frequency word may require selective augmentation by IFG of their activation to reach sufficient familiarity for a lexical decision to be made. In the current study, the speech stimuli were real words that had phonetically sufficient familiarity; on the other hand, they did not have sufficient familiarity when the pitch accents were taken into consideration. Therefore, the significant activation in the IFG in the INCORRECT condition compared with the NORMAL condition might be explained by the effect of familiarity.

Next, we consider the reason why significant activation in the right IFG was observed in both conditions. In Xiao et al.'s experiments [10], participants carried out an auditory lexical decision task based on real and pseudo Chinese words. As a result, the bilateral IFG was more highly activated for pseudo-words than for real words. They discussed the increased activation in this area for pseudo-words and attributed it to differences in the decision-making processes. Carreiras et al. [11] compared the neural implementation of word and pseudo-word processing during two commonly used word recognition tasks: lexical decision-making and reading aloud. They showed that increased activation for pseudo-words relative to real words was greater in the right IFG for lexical decision-making than for reading. They discussed how the participants must inhibit word responses to wordlike pseudo-words when making lexical decisions for pseudowords. The activation of the IFG under the PSEUDO condition in the current study seems to agree well with their suggestion.

On the other hand, the significant activation in the right IFG under the INCORRECT condition could not be explained by cognitive inhibition because the participants made a lexical decision by giving the response "word" when they heard a word with incorrect accents. Wong et al. [29] investigated the difference in brain activation between native Mandarin-speaking and English-speaking listeners. As a result, when Mandarin-speaking participants discriminated the same pitch patterns embedded in English words, activations were primarily in the right hemisphere, including the IFG, which has been shown to be activated during pitch processing. On the other hand, when Englishspeaking participants discriminated pitch patterns of English word pairs without a lexical function, the right hemisphere, including the right IFG, was strongly activated. According to their results, the right IFG might serve in nonlexical pitch processing. If the activation in the right IFG observed in the current experiment can be interpreted as being brought about by nonlexical pitch processing, pitch patterns of the words with incorrect accents might be processed as nonlexical information.

\subsection{Superior Temporal Gyrus}

In the INCORRECT condition compared with the NORMAL condition, significant activation in the right STG was observed. The STG is considered to be a major 
region for prosody processing [23], and several studies on the relation between the STG, and prosody [21,22,24,25], and pitch processing [1] have been reported.

\subsection{Superior Part of Ventral Premotor Area}

For the PMsv, significant activation was observed bilaterally in the INCORRECT condition compared with the NORMAL condition, and observed on the right side in the PSEUDO condition compared with the NORMAL condition.

Several researchers consider the ventral premotor area to be concerned with the planning of movement [30-32]. Moreover, even in the case of passive speech perception, activation in the region was observed. Wilson and Iacoboni [20] observed neural responses to unfamiliar non-native phonemes varying in the extent to which they can be articulated. Both superior temporal (auditory) and precentral (motor) areas were activated by passive speech perception, and both distinguished non-native from native phonemes with a greater signal change in response to nonnative phonemes. According to their discussion, superior temporal auditory areas are bilaterally crucial for the transformation of acoustic speech input to a phonetic code. The posterior superior temporal plane in particular matched the auditory input to stored templates. Motor areas were functionally connected to the superior temporal cortex. When hearing a non-native phoneme, a match is never obtained, so the motor system is engaged in repeated attempts to generate other internal auditory templates, leading to greater motor activity.

According to Wilson and Iacoboni's suggestion, one possible interpretation of activations in the PMsv in the current experiment might be related to generation of other internal auditory templates. When the participants detected incorrect pitch accents in the INCORRECT condition or an unprecedented sequence of syllables in the PSEUDO condition, other internal auditory objects might be generated, and repeated silent rehearsal [28] might be performed to identify the word.

\subsection{Left Supplementary Motor Area}

As a result of the contrast between INCORRECT and NORMAL observed in the current study, significant activation in the left SMA was observed. The left SMA was reported to be involved in several functions for word recognition, such as the lexico-phonological process [13], internal specification of verbal responses [30], and an effortful word selection process [31]. The regions of the precentral and SMA would also be related to the initiation of a speech plan in speech production [31]. Although the functional attributes of the SMA are complex [16,33], if we note that the SMA is concerned with motor planning, the activation observed in SMA can be interpreted as support- ing silent rehearsal together with the activation observed in the promoter area. Activation in the left SMA and PMsv in the current study is consistent with the suggestion that speech perception is a sensory-motor process $[19,20,28,34-36]$.

\subsection{Conclusion}

In this study, brain activities when listening to spoken Japanese with incorrect pitch accents compared with normal accents, were investigated. The results indicated that real words with incorrect pitch accents contrasted to those with normal pitch accent cause significant activation in several regions: the bilateral inferior frontal gyrus (IFG), the left supplementary motor area (SMA), the bilateral superior part of the ventral premotor area (PMsv), and the right superior temporal gyrus.

When the data are combined with results from previous studies, we conjectured the characteristic processing when a real word with an incorrect accent is presented as an auditory stimulus to be as follows: when a pitch accent of the stimulus does not match the template, an inner template may be generated and compared recursively to resolve the mismatch and identify the word. Such a silent rehearsal might lead to activation in the premotor area. To obtain the pitch pattern necessary for generating internal speech, nonlexical pitch processing may be needed, which might lead to activation in the right IFG. The STG, which might play a dominant role in the first stage of prosody processing, might be active in the recursive sensory-motor process including inner template generation, silent rehearsal, and lexical decision.

\section{ACKNOWLEDGEMENTS}

The fMRI experiment was performed with technical support from the ATR Brain Activity Imaging Center (BAIC). The author thanks Dr. Yukiko Nota and Dr. Takanori Kochiyama for instructive comments as well as Mr. Taichi Shikata for his assistance in the experiments. The present author was able to use the STRAIGHT system due to the courtesy of Dr. Hideki Kawahara.

\section{REFERENCES}

[1] P. C. M. Wong, T. K. Perrachione and T. B. Parrish, "Neural characteristics of successful and less successful speech and word learning in adults," Hum. Brain Mapp., 28, 995-1006 (2007).

[2] I. Masuda-Katsuse, "Contribution of pitch-accent information to Japanese spoken-word recognition," Acoust. Sci. \& Tech., 27, 97-103 (2006).

[3] S. Amano and T. Kondo, Lexical Properties of Japanese (Sanseido, Tokyo 1999).

[4] H. Kawahara, I. Masuda-Katsuse and A. de Cheveigné, "Restructuring speech representations using a pitch-adaptive time-frequency smoothing and an instantaneous-frequency- 
based F0 extraction: Possible role of a repetitive structure in sounds," Speech Commun., 27, 187-207 (1999).

[5] A. V. Oppenheim and R. W. Schafer, Homomorphic Signal Processing in Digital Signal Processing (Prentice-Hall, Englewood Cliffs, N.J., 1975).

[6] H. Dunn and S. White, "Statistical measurements on conversational speech," J. Acoust. Soc. Am., 11, 278-288 (1940).

[7] J. Wu, C. Cai, T. Kochiyama and K. Osaka, "Function segregation in the left inferior frontal gyrus: a listening functional magnetic resonance imaging study," NEUROREPORT, 18, 127-131 (2007).

[8] C. Cai, T. Kochiyama, K. Osaka and J. Wu, "Lexical/semantic processing in dorsal left inferior frontal gyrus," NEUROREPORT, 18, 1147-1151 (2007).

[9] S. D. Newman and D. Twieg, "Differences in auditory processing of words and pseudowords: An fMRI study," Hum. Brain Mapp., 14, 39-47 (2001).

[10] Z. Xiao, J. X. Zhang, X. Wang, R. Wu, X. Hu, X. Weng and L. H. Tan, "Differential activity in left inferior frontal gyrus for pseudowords and read words: An event-related fMRI study on auditory lexical decision," Hum. Brain Mapp., 25, 212-221 (2005).

[11] M. Carreiras, A. Mechelli, A. Estévez and C. J. Price, "Brain activation for lexical decision and reading aloud: Two sides of the same coin?," J. Cognit. Neurosci., 19, 433-444 (2007).

[12] J. A. Fiez, D. A. Balota, M. E. Raichle and S. E. Petersen, "Effects of lexicality, frequency, and spelling-to-sound consistency on the functional anatomy of reading," Neuron, 24, 205-218 (1999).

[13] M. Carreiras, A. Mechelli and C. J. Price, "Effect of word and syllable frequency on activation during lexical decision and reading aloud," Hum. Brain Mapp., 27, 963-972 (2006).

[14] M. Nakic, B. W. Smith, S. Busis, M. Vythilingam, R. James and R. Blair, "The impact of affect and frequency on lexical decision: The role of the amygdala and inferior frontal cortex," NeuroImage, 31, 1752-1761 (2006).

[15] K. R. Ridderinkhof, S. Nieuwenhuis and T. S. Braver, "Medial frontal cortex function: An introduction and overview," Cognit. Affect. Behav. Neurosci., 7, 261-265 (2007).

[16] P. Nachev, C. Kennard and M. Husain, "Functional role of the supplementary and pre-supplementary motor areas," Nat. Rev. Neurosci., 9, 856-869 (2008).

[17] C. Wang, I. Ulbert, D. L. Schomer, K. Marinkovic and E. Halgren, "Responses of human anterior cingulate cortex microdomains to error detection, conflict monitoring, stimulus-response mapping, familiarity, and orienting," J. Neurosci., 25, 604-613 (2005).

[18] H. Lütcke and J. Frahm, "Lateralized anterior cingulate function during error processing and conflict monitoring as revealed by high-resolution fMRI," Cereb. Cortex, 18, 508515 (2008).

[19] M. Iacoboni, "The role of premotor cortex in speech perception: Evidence from fMRI and rTMS," J. Physiol. Paris, 102, 31-34 (2008).

[20] S. M. Wilson and M. Iacoboni, "Neural responses to nonnative phonemes varying in producibility: Evidence for the sensorimotor nature of speech perception," NeuroImage, 33, 316-325 (2006).

[21] J. Gandour, D. Wong, M. Dzemidzic, M. Lowe, Y. Tong and $\mathrm{X}$. Li, "A cross-linguistic fMRI study of perception of intonation and emotion in Chinese," Hum. Brain Mapp., 18, 149-157 (2003).

[22] C. Humphries, T. Love, D. Swinney and G. Hickok, "Response of anterior temporal cortex to syntactic and prosodic manipulations during sentence processing," Hum. Brain Mapp., 26, 128-138 (2005).

[23] A. K. Ischebeck, A. D. Friederici and K. Alter, "Processing prosodic boundaries in natural and hummed speech: An fMRI study," Cereb. Cortex, 18, 541-552 (2008).

[24] G. Dogil, H. Ackermann, W. Grodd, H. Haider, H. Kamp, J. Mayer, A. Riecker and D. Wildgruber, "The speaking brain: A tutorial introduction to fMRI experiments in the producition of speech, prosody and syntax," J. Neurolinguist., 15, 59-90 (2002).

[25] M. Meyer, K. Steinhauer, K. Alter, A. D. Friederici and D. Y. von Cramon, "Brain activity varies with modulation of dynamic pitch variance in sentence melody," Brain Lang., 89, 277-289 (2004).

[26] M. Brett, J. L. Anton, R. Valabregue and J. B. Poline, "Region of interest analysis using an SPM toolbox [abstract]," Presented at the 8th International Conference on Functional Mapping of the Human Brain, June 2-6, 2002, Sendai, Japan. Available on CD-ROM in NeuroImage, 16, Abstract 497 (2002).

[27] F. A. Nielsen, "The Brede database: a small database for functional neuroimaging," NeuroImage, 19, Presented at the 9th International Conference on Functional Mapping of the Human Brain, 19-22 (2003).

[28] M. Vigneau, V. Beaucousin, P. Y. Hervé, H. Duffau, F. Crivello, O. Houdé, B. Mazoyer and N. Tzourio-Mazoyer, "Meta-analyzing left hemisphere language areas: Phonology, semantics, and sentence processing," NeuroImage, 30, 14141432 (2006).

[29] P. C. M. Wong, L. M. Parsons, M. Martinez and R. L. Diehl, "The role of the insular cortex in pitch pattern perception: The effect of linguistic contexts," J. Neurosci., 24, 9153-9160 (2004).

[30] P. Tremblay and V. L. Gracco, "Contribution of the frontal lobe to externally and internally specified verbal responses: fMRI evidence," NeuroImage, 33, 947-957 (2006).

[31] J. W. Bohland and F. H. Guenther, "An fMRI investigation of syllable sequence production," NeuroImage, 32, 821-841 (2006).

[32] S. R. Simon, M. Meunier, L. Piettre, A. M. Berardi, C. M. Segebarth and D. Boussaoud, "Spatial attention and memory versus motor preparation: Premotor cortex involvement as revealed by fMRI," J. Neurophysiol., 88, 2047-2057 (2002).

[33] F.-X. Alario, H. Chainay, S. Lehericy and L. Cohen, "The role of the supplementary motor area (SMA) in word production," Brain Res., 1076, 129-143 (2006).

[34] G. Hickok and D. Poeppel, "Towards a functional neuroanatomy of speech perception," Trends Cognit. Sci., 4, 131138 (2000).

[35] I. G. Meister, S. M. Wilson, C. Deblieck, A. D. Wu and M. Iacoboni, "The essential role of premotor cortex in speech perception," Curr. Biol., 17, 1692-1696 (2007).

[36] R. Jardri, D. Pins, M. Bubrovszky, P. Despretz, J.-P. Pruvo, M. Steinling and P. Thomas, "Self awareness and speech processing: An fMRI study," Neurolmage, 35, 1645-1653 (2007). 